Bundesgesundheitsbl 2019 - 62:612-619 https://doi.org/10.1007/s00103-019-02930-0 Online publiziert: 28. März 2019

(C) Der/die Autor(en) 2019, korrigierte Publikation 2019

\section{Zwischen Wetter und Klimawandel}

Der Sommer 2018 dürfte vielen Menschen in Deutschland in Erinnerung bleiben - wenn auch aus unterschiedlichen Gründen. Während die Landwirtschaft durch die anhaltende Hitze und Trockenheit Ernteausfälle zu beklagen hatte [1], wurden Ventilatoren landesweit zur Mangelware [2] und die Speiseeisindustrie legte Sonderschichten ein [3]. Angesichts der anhaltenden Wetterextreme [4] wurde die Frage laut, inwiefern diese mit dem menschengemachten Klimawandel zusammenhängen [5].

Zwar können einzelne Wetterereignisse nicht auf den Klimawandel zurückgeführt werden, allerdings erhöht der Klimawandel die Häufigkeit von Extremwetterereignissen [6]. So treten seit 1950 Tropennächte (entsprechen einer maximalen Tiefsttemperatur von $20^{\circ} \mathrm{C}$ ) oder Hitzetage mit Höchstwerten von über $30^{\circ} \mathrm{C}$ deutlich häufiger auf, wohingegen die Zahl der Frosttage (Tiefsttemperatur unter $0^{\circ} \mathrm{C}$ ) abnahm [6]. Auch bei den Niederschlägen zeichnen sich erste Veränderungen ab: Es regnet in Deutschland im Sommer tendenziell weniger, während die Niederschläge in den Wintermonaten zunehmen [7]. Dennoch gibt es Personen, die an der Existenz des menschengemachten Klimawandels zweifeln, wie es zum Beispiel an dem Ausstieg der USA aus dem Pariser Klimaabkommen
Natalie Berger · Ann-Kathrin Lindemann · Gaby-Fleur Böl

Abteilung Risikokommunikation, Bundesinstitut für Risikobewertung (BfR), Berlin, Deutschland deutlich wird [8]. Diese Zweifel werden auch dadurch gefördert, dass kurzfristige ökonomische Interessen (wie die Nutzung fossiler Brennstoffe statt erneuerbarer Energie) zum Teil höher bewertet werden als die langfristigen Folgen des Klimawandels [9, 10]. In einer repräsentativen Bevölkerungsumfrage des Bundesinstituts für Risikobewertung (BfR) war die Klimawandelskepsis in Deutschland dagegen nicht weitverbreitet (siehe - Infobox 1 für methodische Details und - Tab. 1 für die Beschreibung der Stichprobe). So widersprachen nur 3\% der Befragten der Aussage, dass der Klimawandel tatsächlich stattfindet. Mehr als die Hälfte (55\%) stimmte der Aussage dagegen voll und ganz zu.

Da sich die veränderten Klimabedingungen auch auf die Gesundheit der in Deutschland lebenden Bevölkerung auswirken können, stellt der Klimawandel eine Herausforderung für den Gesundheitsschutz dar. Die Bevölkerung sollte von den entsprechenden Behörden und Einrichtungen so auf den Klimawandel vorbereitet werden, dass sie kompetent mit veränderten klimatischen Bedingungen umgehen kann. Dieser Ansatz wird in der Literatur unter dem Begriff „Preparedness“ diskutiert [11]. Im Folgenden stellen wir die erwarteten gesundheitlichen Auswirkungen des Klimawandels dar und gehen auf die sich daraus ergebenden Konsequenzen für die Risikokommunikation ein.

\section{Auswirkungen des Klima- wandels auf die menschliche Gesundheit}

Aus den mit dem Klimawandel einhergehenden Wetter- und Umweltveränderungen lassen sich schon jetzt direkte und indirekte Auswirkungen auf die Gesundheit der in Deutschland lebenden Bevölkerung ableiten. ${ }^{1}$ Die direkten physischen oder psychischen gesundheitlichen Auswirkungen werden unmittelbar von den Temperatur- bzw. Wetteränderungen verursacht. Indirekte Wirkungen hingegen werden von Prozessen verursacht, die selbst erst durch Temperaturund Wetteränderungen ausgelöst werden [7].

$\mathrm{Zu}$ den direkten gesundheitlichen Auswirkungen zählt die Zunahme von Toten und Verletzten durch Hitzewellen, Starkregenereignisse, Stürme, Erdrutsche und Lawinen [7]. Zwar stellen diese Wetterextreme auch ungeachtet des Klimawandels ein gesundheitliches Risiko dar, allerdings ist davon auszugehen, dass sich die Auswirkungen aufgrund einer steigenden Häufigkeit von Extremwetterereignissen weiter verstärken werden [12]. So ist bekannt, dass Hitzewellen beispielsweise aufgrund ihrer belastenden Wirkung auf das Herz-KreislaufSystem die Sterblichkeit insbesondere

\footnotetext{
1 Eine aktuelle Übersicht zu den globalen gesundheitlichen Auswirkungen findet sich im letzten Special Report des Intergovernmental Panel on Climate Change (IPCC) der Vereinten Nationen [12].
} 


\section{Infobox 1 Methodische Details der Bevölkerungsbefragung}

Im Auftrag des BfR wurden 1018 Personen von einem Marktforschungsinstitut mittels einer computergestützten Telefonbefragung zum Thema Klimawandel und den damit verbundenen gesundheitlichen Risiken befragt. Im Rahmen einer Omnibus-Erhebung wurden deutschsprachige Personen ab 14 Jahren im Zeitraum vom 24.09. bis 01.10.2018 interviewt. Junge und niedrig gebildete Personen waren in der Stichprobe unterrepräsentiert. Um die Repräsentativität der Stichprobe zu gewährleisten, wurde sie durch Gewichtung an die demografischen Strukturen der Grundgesamtheit angepasst. Grundlage dafür waren die aktuelle Bevölkerungsfortschreibung und der aktuelle Mikrozensus des Statistischen Bundesamtes. Die demografische Struktur der finalen Stichprobe ist in - Tab. 1 dargestellt. Die statistische Fehlertoleranz der Ergebnisse lag bei maximal $\pm 3,1$ Prozentpunkten. Die Befragung dauerte im Durchschnitt 4,5 min. Die erste Frage, ob die Befragten der Aussage zustimmten, dass der Klimawandel tatsächlich stattfindet, diente als Filter. Personen, die diese Frage mit "stimme gar nicht zu" beantworteten (3\%), sowie jene, die keine Antwort wussten bzw. keine Angabe machen wollten ( $1 \%$ ), wurden von der weiteren Befragung ausgeschlossen. ${ }^{*}$ Die verbleibenden 972 Personen nahmen an der gesamten Befragung teil.

* Es kann nicht ausgeschlossen werden, dass Skepsis gegenüber dem Klimawandel in Befragungen unterschätzt wird, da Personen ihre Skepsis nicht offen äußern möchten (soziale Erwünschtheit).

von älteren Personen und Kleinkindern erhöhen können [7]. In Folge von Ereignissen wie Überschwemmungen, Lawinen oder Waldbränden können bei einigen Überlebenden zudem psychische Probleme wie posttraumatische Belastungsstörungen auftreten [6]. Eine zunehmende Zahl an Sonnenstunden kann zudem die UV-Belastung erhöhen und vermehrt zu Hautkrebs und Augenlinsentrübungen führen [13]. Durch die Sonneneinstrahlung gebildetes bodennahes Ozon stellt durch die Reizung der Atemwege insbesondere für Personen mit Atemwegserkrankungen ein gesundheitliches Risiko dar [14].

Die indirekten gesundheitlichen Auswirkungen lassen sich grob in zwei Kategorien einteilen: die Ausbreitung von fremden, für den Menschen potenziell gefährlichen Pflanzen- und Tierarten sowie die Ausbreitung von Krankheitserregern [7]. Wenn sich bislang gebietsfremde Pflanzen- und Tierarten in einem für sie neuen Gebiet ausbreiten, so kann dies unter anderem das Allergierisiko erhöhen [14]. Ein bekanntes Beispiel ist die Beifuß-Ambrosie (Ambrosia artemisiifolia). Diese Pflanze kann nicht nur Kontaktallergien auslösen, sondern verlängert als Spätblüher auch die saisonale Pollenbelastung [14]. Auch Tierarten können das Allergiepotenzial erhöhen. Durch die fortschreitende Ausbreitung des Eichenprozessionsspinners (Thaumetopoea processionea) beispielsweise können vermehrt Menschen mit den Brennhaaren der Raupe in Kontakt kommen, welche gesundheitliche Beeinträchtigungen hervorrufen können, angefangen bei Hautirritationen bis hin zu Atemproblemen [16]. Zusätzlich können veränderte klimatische Bedingungen die Konzentration von unerwünschten Pflanzeninhaltsstoffen beeinflussen. Ein Beispiel hierfür sind Pyrrolizidinalkaloide (PA), die Pflanzen zum Schutz vor Fraßfeinden bilden. In hoher Dosierung können PA akut zu Leberschädigungen beim Menschen führen [15].

Die Ausbreitung von Krankheitserregern kann durch den Klimawandel auf verschiedene Weise befördert werden. So können Insekten als sogenannte Vektoren fungieren und für den Menschen gefährliche Krankheiten übertragen [14]. Wenn sich durch wärmeres Wetter in Deutschland Insekten ausbreiten, die Tropenkrankheiten wie Denguefieber oder Malaria übertragen, könnte dies $\mathrm{zu}$ einer stärkeren Verbreitung dieser Krankheiten führen. Allerdings müssten die Insekten dafür bereits mit den Erregern infiziert sein. In Mitteleuropa treten diese Krankheiten jedoch bislang kaum auf, weswegen weitere Infektionen durch Insekten derzeit unwahrscheinlich sind [6,7]. Wetterveränderungen können jedoch auch die Populationen von heimischen Vektoren wie Zecken beeinflussen und damit die Verbreitung von bereits bekannten Krankheiten (z. B. Lyme-Borreliose) erhöhen [7].

Auch die Infektionszahlen bei Krankheiten, die über Lebensmittel oder Was- ser übertragen werden, können ansteigen. Höhere Temperaturen begünstigen die Ausbreitung von Krankheitserregern wie Vibrionen oder Cyanobakterien („Blaualgen“) in Seen und Flüssen [17]. Bei warmem Wetter breiten sich auch Lebensmittelkeime wie Salmonellen und Campylobacter stärker aus, weshalb auch hier mit höheren Erkrankungszahlen zu rechnen ist. Bei anhaltend hohen Temperaturen werden zudem vermehrt Lebensmittel wie Grillfleisch, Speiseeis oder Frischeiprodukte konsumiert, welche als Risikoprodukte für eine Lebensmittelinfektion bekannt sind [7]. In Lebensmitteln können sich bei höheren Temperaturen zudem giftige Stoffe anreichern, wie beispielsweise Algentoxine in Muscheln [7].

Der Klimawandel kann sich vereinzelt auch positiv auf die menschliche Gesundheit auswirken. Ein milderer Winter kann beispielsweise die Wintermortalität eindämmen und häufigeres sonniges Wetter die Vitamin D-Versorgung der Bevölkerung verbessern [7]. Allerdings überwiegen die gesundheitlichen Risiken diese möglichen positiven Folgen deutlich.

\section{Konsequenzen für die Risikokommunikation}

Damit die Bevölkerung adäquat auf die veränderten klimatischen Bedingungen reagieren kann, sollte sie angemessen auf die damit einhergehenden Risiken vorbereitet werden. Eine wichtige Maßnahme ist dabei die Entwicklung von konkreten Handlungsplänen. Beispiele hierfür sind die Handlungsempfehlungen des Robert Koch-Instituts und des Umweltbundesamts zum Thema Klimawandel und Gesundheit [13] oder die Handlungsempfehlungen des Bundesministeriums für Umwelt, Naturschutz und nukleare Sicherheit für die Entwicklung von Hitzeaktionsplänen [18]. Diese Publikationen bieten eine Orientierung bei der Frage, wie verschiedene Stakeholder über geeignete Präventions- oder Risikovermeidungsmaßnahmen informiert werden können. Ein wichtiges Element sind dabei die Identifikation und Definition von vulnerablen sowie schwer erreichbaren Zielgruppen. Es müssen Kommunikationsmaßnahmen 
entwickelt werden, welche nicht nur auf die Bedürfnisse einer vulnerablen Zielgruppe zugeschnitten sind, sondern auch Personen erreichen, die z. B. nicht über massenmediale Kanäle angesprochen werden können. Dabei können auch Multiplikatoren wie Lehrkräfte oder Ärztinnen und Ärzte eingebunden werden. Nur wenn klar definiert ist, welche Gruppen von einem bestimmten Risiko betroffen sind und über welche Kanäle man diese erreichen kann, lassen sich geeignete Risikokommunikationsmaßnahmen für die Bedürfnisse der Zielgruppe entwickeln [19]. Ein Beispiel hierfür sind Aus- und Weiterbildungsformate zu Aspekten wie UVund Hitzeschutz für Berufsgruppen, die regelmäßig unter freiem Himmel arbeiten.

Im Rahmen der Risikokommunikation $\mathrm{zu}$ den gesundheitlichen Auswirkungen des Klimawandels ist außerdem zu beachten, dass sich schon bestehende Themen wandeln oder eine Themenverschiebung stattfindet. So sind Allergien als Thema in der Risikokommunikation bereits bekannt. Allerdings müssen zukünftig auch neue Allergene oder eine längere Pollenflugsaison berücksichtigt werden. Dabei geht es nicht nur darum, das Bewusstsein für bestimmte Risikofaktoren zu stärken, sondern auch Verhaltensänderungen herbeizuführen. Wenn durch wärmere Temperaturen das Risiko für Lebensmittelinfektionen steigt, sollte die Risikokommunikation darauf adäquat reagieren und einen stärkeren Fokus auf Verhaltensweisen wie eine angemessene Küchenhygiene im Privathaushalt legen. Eine Ausbreitung von krankmachenden Keimen in Gewässern könnte zudem dazu führen, dass Essgewohnheiten (wie der regelmäßige Verzehr von Meerestieren) verändert werden müssen. Die Herausforderung für die Risikokommunikation besteht somit darin, etablierte Gewohnheiten zu verändern.

Bei der Risikokommunikation sollte auch die Risikowahrnehmung der Bevölkerung berücksichtigt werden. Insbesondere, wenn in der Bevölkerung einzelne gesundheitliche Auswirkungen des Klimawandels wenig Beachtung finden, kann die Risikokommunikation

Bundesgesundheitsbl 2019 • 62:612-619 https://doi.org/10.1007/s00103-019-02930-0 (c) Der/die Autor(en) 2019

N. Berger · A.-K. Lindemann · G.-F. Böl

\section{Wahrnehmung des Klimawandels durch die Bevölkerung und Konsequenzen für die Risikokommunikation}

\section{Zusammenfassung}

Der menschengemachte Klimawandel geht mit einer Reihe von Wetter- und Umweltveränderungen einher, die das Ausmaß bestimmter gesundheitlicher Risiken beeinflussen können. Der Beitrag gibt einen Überblick über die Herausforderungen, die sich bei der Kommunikation über diese gesundheitlichen Risiken ergeben. Dabei werden theoretische Überlegungen mit den Ergebnissen einer repräsentativen Bevölkerungsumfrage in Beziehung gesetzt, in der 1018 Personen ab 14 Jahren zum Thema Klimawandel und zu den damit verbundenen gesundheitlichen Risiken befragt wurden. Laut Umfrageergebnissen ist eine Mehrheit der Bevölkerung davon überzeugt, dass der Klimawandel tatsächlich stattfindet. Die Daten deuten jedoch darauf hin, dass die Befragten dem Thema mit psychologischer Distanz begegnen. Zudem spielen gesundheitliche Risiken im Zusammenhang mit dem Klimawandel in der Wahrnehmung der Befragten eine vergleichsweise untergeordnete Rolle.
Die theoretischen Überlegungen in Verbindung mit den empirischen Ergebnissen verdeutlichen, dass gezielte Kommunikationsmaßnahmen nötig sind, um die Bevölkerung für gesundheitliche Risiken im Zusammenhang mit dem Klimawandel zu sensibilisieren. Dabei bedarf es nicht nur eines umfassenden Informationsangebots, sondern auch Anleitungen und Handlungspläne zur praktischen Umsetzung des Wissens. Kommunikationsmaßnahmen sollten Faktoren berücksichtigen, welche die Risikowahrnehmung sowie die Handlungsbereitschaft beeinflussen können, und in ein strategisches Kommunikationskonzept eingebettet sein, welches die Ansprache unterschiedlicher Zielgruppen ermöglicht.

Schlüsselwörter

Risikowahrnehmung - Public Health . Gesundheit · Kommunikationsstrategien . Repräsentative Bevölkerungsbefragung

\section{Public perception of climate change and implications for risk communication}

\section{Abstract}

Man-made climate change is associated with several weather and environmental changes that can influence the extent of certain health risks. The article gives an overview of the challenges of communicating these health risks. Theoretical considerations are linked with the results of a representative population survey, in which 1018 participants aged 14 years and above were polled about their perception of climate change and its associated health risks.

According to the survey results, the majority of the population is convinced that climate change is actually taking place. However, the data suggest that respondents regard the topic with psychological distance. Health risks associated with climate change also play a relatively minor role in the participants' perception.
The theoretical considerations in connection with the empirical results show that targeted communication measures are needed to enhance awareness of the health risks associated with climate change among the general public. This requires not only a comprehensive provision of information, but also instructions and action plans for the practical implementation of that knowledge. Communication measures should take into account factors that can influence risk perception and the willingness to act. They should also be embedded in a strategic communication concept that allows different target groups to be addressed.

\section{Keywords}

Risk perception - Public health - Health . Communication strategies - Representative survey 
Tab. 1 Demografische Struktur der Bevölkerungsstichprobe $(n=1018)$ in Prozent

\section{Geschlecht}

Weiblich

51,0

Männlich

49,0

Alter

14 bis 19 Jahre

8,9

20 bis 29 Jahre

11,9

30 bis 39 Jahre

14,0

40 bis 49 Jahre

16,0

50 bis 59 Jahre

18,2

60 bis 69 Jahre

13,4

70 Jahre und älter

Bildungsstand

Volks- oder Hauptschule

Weiterbildende Schule, ohne

Abitur

Abitur, Studium

Schülerinnen und Schüler

Ländergebiete

Ost

West

$n=$ Fallzahl

verstärkt über die damit verbundenen Risiken aufklären. Wie in $\bullet$ Tab. 2 deutlich wird, schätzt die deutschsprachige Bevölkerung den Klimawandel allgemein als relativ beunruhigend ein. Bei den spezifischen gesundheitlichen $\mathrm{Ri}$ siken der zu erwartenden Wetter- und Umweltbedingungen sind allerdings geringe Unterschiede in der Risikoeinschätzung erkennbar. Die Befragten sind besonders beunruhigt über die Veränderungen, die bereits heute sicht- und spürbar sind (Hitzewellen, Unwetter und Überschwemmungen). Indirekte Auswirkungen lösen bei den Befragten dagegen weniger starke Beunruhigung aus, obwohl diese für die Gesundheit nicht weniger relevant sind. Die Daten deuten darauf hin, dass bestimmte Risiken für die Bevölkerung eine weniger wichtige Rolle spielen, weshalb diese in der Risikokommunikation eine stärkere Berücksichtigung finden sollten. Allerdings ist zu beachten, dass es sich bei der Befragung um eine Momentaufnahme handelt, die möglicherweise durch vergangene Ereignisse (wie den Hitzesommer 2018) beeinflusst wurde. Die Risikowahrnehmung sollte deshalb nach Möglichkeit wiederholt erhoben wer-

Tab. 2 Beunruhigung über den Klimawandel. Repräsentative Bevölkerungsbefragung mit 1018 Personen in Deutschland im Jahr 2018 (siehe Infobox 1)

\begin{tabular}{|c|c|c|c|}
\hline Frageformulierungen & $M$ & SD & $n$ \\
\hline $\begin{array}{l}\text { Inwieweit sind Sie über den Klimawandel beunruhigt oder nicht } \\
\text { beunruhigt? }\end{array}$ & 3,6 & 1,1 & 966 \\
\hline \multicolumn{4}{|c|}{$\begin{array}{l}\text { Inwieweit sind Sie über die folgenden Risiken, die im Zusammenhang mit dem Klimawandel } \\
\text { diskutiert werden, beunruhigt oder nicht beunruhigt? }\end{array}$} \\
\hline $\begin{array}{l}\text { - Häufigeres Auftreten von Hitzewellen, Dürren und Waldbrän- } \\
\text { den }\end{array}$ & 4,1 & 1,0 & 968 \\
\hline - Stärkere Belastung von Gewässern mit Krankheitserregern & 3,6 & 1,1 & 953 \\
\hline - Stärkere Belastung von Lebensmitteln mit Krankheitserregern & 3,3 & 1,3 & 959 \\
\hline $\begin{array}{l}\text { - Ausbreitung allergieauslösender Pflanzenarten (z. B. Ambrosia) } \\
\text { in neue Gebiete }\end{array}$ & 3,3 & 1,2 & 937 \\
\hline $\begin{array}{l}\text { - Ausbreitung allergieauslösender Tierarten (z. B. Eichenprozes- } \\
\text { sionsspinner) in neue Gebiete }\end{array}$ & 3,3 & 1,2 & 945 \\
\hline $\begin{array}{l}\text { - Ausbreitung von Insekten, die Krankheitserreger in sich tra- } \\
\text { gen, in neue Gebiete }\end{array}$ & 3,6 & 1,1 & 961 \\
\hline $\begin{array}{l}\text { - Häufigeres Auftreten von Unwettern und Überschwemmun- } \\
\text { gen }\end{array}$ & 4,1 & 1,0 & 966 \\
\hline - Höhere Schadstoffkonzentration in der Atemluft (z. B. Ozon) & 3,6 & 1,2 & 956 \\
\hline
\end{tabular}

den, um den Einfluss situativer Faktoren besser abschätzen zu können.

Für die Umsetzung von gezielten Kommunikationsmaßnahmen sollten weiterhin konkrete Herausforderungen für die Risikokommunikation zum Thema Klimawandel beachtet werden [20]. Ein Beispiel hierfür ist die wissenschaftliche Unsicherheit in der Prognose von Klimaveränderungen, die sowohl für die Risikowahrnehmung als auch für die Handlungsbereitschaft in der Bevölkerung eine Rolle spielen kann. Im Folgenden werden die zentralen Herausforderungen bei der Kommunikation über die gesundheitlichen Auswirkungen des Klimawandels dargelegt sowie Empfehlungen für den Umgang mit diesen gegeben.

\section{Wahrnehmung gesundheit- licher Risiken im Zusammen- hang mit dem Klimawandel}

Die Herausforderungen für die Kommunikation über gesundheitliche Risiken im Zusammenhang mit dem Klimawandel sind untrennbar mit denen verbunden, die für die Kommunikation über den Klimawandel im Allgemeinen gelten. Zu den größten Herausforderungen zählt, dass der Klimawandel bzw. die ihn verursachenden Treibhausgase nicht sichtbar und nicht mit unmittelbaren gesundheitlichen Folgen verbunden sind [7, 20]. Hinzu kommt, dass der Mensch in der modernen, westlichen Gesellschaft sowohl direkt (z. B. durch Gebäudedämmung, Klimaanlagen) als auch indirekt (z. B. durch Versicherungen gegen Unwetterschäden) vor Umwelteinflüssen und ihren Folgen geschützt ist [20, 21], was die Annahme befördern könnte, auch vor gesundheitlichen Risiken durch Umwelteinflüsse sicher zu sein.

Nicht zuletzt prägen auch die Komplexität des Themas und die wissenschaftliche Unsicherheit, die im Zusammenhang mit dem Klimawandel diskutiert wird, die Wahrnehmung gesundheitlicher Risiken. Klimawandelskeptiker machen sich wissenschaftliche Unsicherheit zunutze, um Erkenntnisse zum Klimawandel infrage zu stellen [22-24]. Auch haben empirische Studien zeigen können, dass wahrgenommener wissenschaftlicher Dissens mit einer geringeren Überzeugung, dass der Klimawandel stattfindet, assoziiert ist sowie mit einer geringeren Akzeptanz gegenüber den damit ver- 


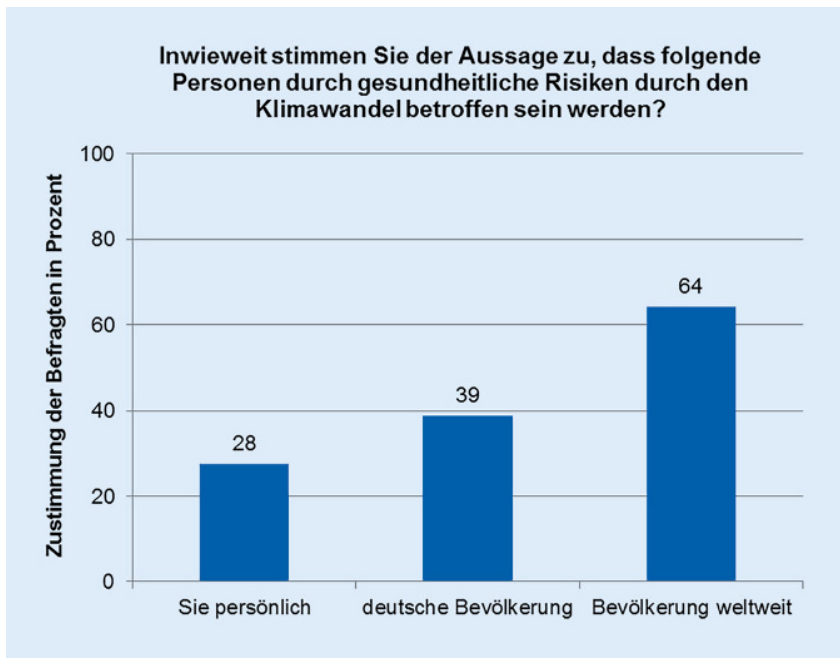

Abb. $1<$ Prozentueller Anteil der Zustimmung (Skalenwerte 4 und 5) unter den Befragten, dass bestimmte Personen von gesundheitlichen Risiken durch den Klimawandel betroffen sein werden $(n=972)$. Skala von $1={ }_{\text {"stimme }}$ überhaupt nicht zu" bis $5={ }_{\text {,stimme voll }}$ und ganz zu"

bundenen Forschungsbemühungen und Eindämmungsmaßnahmen [25, 26]. Da wissenschaftliche Unsicherheit vor allem bezogen auf Ausmaß und Folgen des Klimawandels diskutiert wird [20], könnten gesundheitliche Risiken durch den Klimawandel auf dieser Basis ebenfalls angezweifelt werden. Unsicherheit in der Folgenabschätzung bedeutet aber auch, dass die Folgen schwerwiegender ausfallen können als angenommen. Um dieser Herausforderung zu begegnen, sollten Kommunikationsmaßnahmen gesundheitliche Risiken im Zusammenhang mit dem Klimawandel veranschaulichen und den Fokus auf bereits sicht- und wahrnehmbare gesundheitliche Folgen lenken.

Die Notwendigkeit einer zielgerichteten Risikokommunikation zu gesundheitlichen Folgen des Klimawandels ist jedoch auch dann gegeben, wenn in der Bevölkerung kein Zweifel am Klimawandel und seinen Folgen besteht. In der Literatur wird berichtet, dass auch Personen, die von der Existenz des menschengemachten Klimawandels überzeugt sind, dem Phänomen mit "psychologischer Distanz" begegnen können [27, 28]. Unter diesem Konstrukt ist die Wahrnehmung zu verstehen, dass ein Objekt oder Ereignis zeitlich oder räumlich weit entfernt, die Eintrittswahrscheinlichkeit niedrig oder die Relevanz für die eigene Person bzw. das eigene soziale Netzwerk gering ist [29]. So deutet auch das Antwortverhalten in der hier gezeigten Bevölkerungsumfrage des
BfR auf psychologische Distanz der Befragten hin, von denen die meisten der Aussage zustimmten, dass die Weltbevölkerung von gesundheitlichen Risiken im Zusammenhang mit dem Klimawandel betroffen sein wird. Der Anteil der Zustimmung sank jedoch für die gleiche Aussage bezogen auf die deutsche Bevölkerung und war am niedrigsten bezogen auf die eigene Person (siehe • Abb. 1).

Umgekehrt können Personen durchaus Risiken für Bevölkerungsgruppen sehen, denen sie zugeordnet werden, denen sie sich jedoch nicht als zugehörig empfinden. So zeigte eine Studie zur Wahrnehmung gesundheitlicher Risiken während Hitzeperioden, dass ältere Personen zwischen 72 und 94 Jahren sich selbst weder als "vulnerabel“ noch als „alt" einschätzten, obwohl sie Alter durchaus mit größeren gesundheitlichen Risiken durch Hitzewellen in Verbindung brachten [30]. Auch wenn sie sich bewusst waren, dass bestimmte Krankheiten mit erhöhter Vulnerabilität bei Hitze einhergehen, sahen sie diesen Zusammenhang trotz vorliegender Erkrankung nur für andere, aber nicht für sich selbst. Da psychologische Distanz auch im $\mathrm{Zu}$ sammenhang mit dem Klimawandel mit einer reduzierten Handlungsbereitschaft einhergehen kann [28], sollten Kommunikationsstrategien einen direkten Bezug zu den relevanten Zielgruppen herstellen, um dem Phänomen der psychologischen Distanz zu begegnen. Bei der Ansprache bestimmter Zielgruppen kann es dabei nützlich sein, den Inter- pretationsspielraum $\mathrm{zu}$ begrenzen: So könnte die sehr allgemein angegebene Zielgruppe der „älteren Menschen“ je nach eigenem Alter verschieden interpretiert und damit als für die eigene Person unzutreffend empfunden werden. Die genauer definierte Zielgruppe der "Menschen ab 65 Jahre“ oder der "Generation 65+" lässt dagegen weniger Interpretationsspielraum zu. Dies wurde beispielsweise im "Hitzeknigge“ des Umweltbundesamtes berücksichtigt, in dem Tipps zum richtigen Verhalten bei Hitze gegeben und gezielt Menschen der Generation 65+ angesprochen werden [31].

Eine weitere Herausforderung für die Risikokommunikation ist, die Bedeutung gesundheitlicher Risiken in der Wahrnehmung der Bevölkerung stärker hervorzuheben. Unter den explizit genannten Risiken im Zusammenhang mit dem Klimawandel rangierten in der vorliegenden Umfrage gesundheitliche Risiken zusammen mit sozialen und wirtschaftlichen Folgen für den Menschen an fünfter Stelle (siehe - Abb. 2). Dies könnte darauf hindeuten, dass gesundheitliche Risiken in der Wahrnehmung der Bevölkerung eine vergleichsweise untergeordnete Rolle spielen bzw. von anderen Risiken des Klimawandels überlagert werden. Tatsächlich deuten Studien darauf hin, dass die Auswirkungen des Klimawandels auf die menschliche Gesundheit allgemein unterschätzt werden $[32,33]$. So bezifferten Befragte im Jahr 2005 die Anzahl der jährlich durch die globale Erderwärmung verursachten Todes-, Krankheitsund Verletzungsfälle im Hunderterbereich [33] - eine Schätzung, die weit unter den von der WHO im Jahr 2004 geschätzten 150.000 lag [34]. Ein Großteil der Befragten (40\%) gab an, gar keine Schätzung abgeben zu können. Konkret nach klimawandelbedingten Gesundheitsrisiken gefragt, haben in einer anderen Studie nur $22 \%$ spontan Atemwegserkrankungen nennen können, die als gesundheitliche Folge im Zusammenhang mit dem Klimawandel häufig diskutiert werden [32]. Auch gibt es Themen, die Personen bereits geläufig sind, die sie aber nicht mit dem Klimawandel in Verbindung bringen. Dazu zählen beispielsweise gesundheitliche Belastungen durch Pollen- 
Welche Risiken sehen Sie im Zusammenhang mit dem Klimawandel?

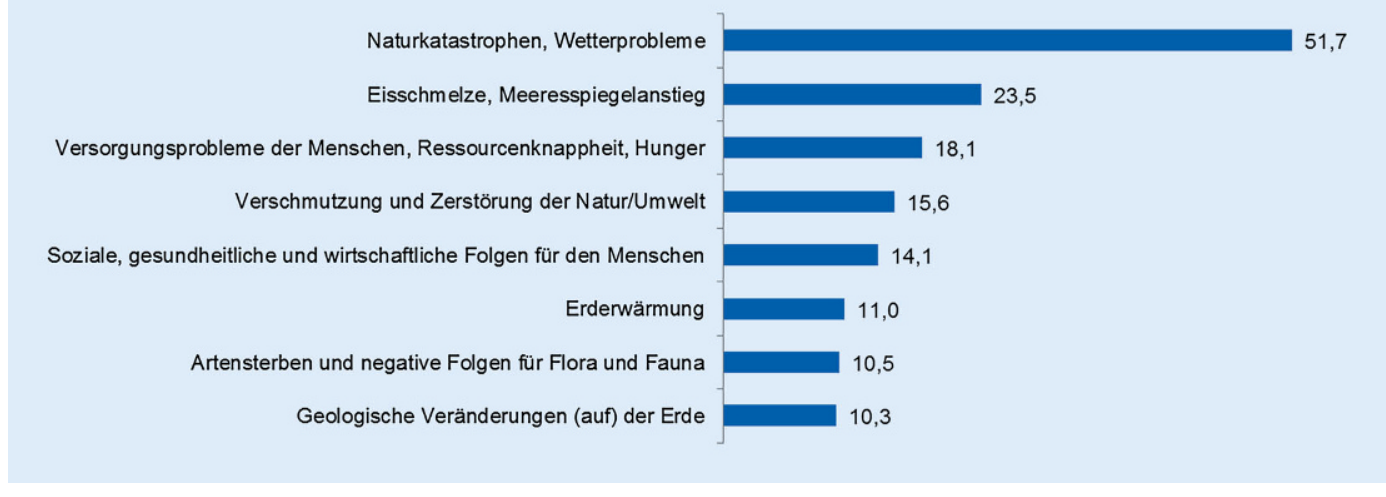

Abb. $2 \triangleleft$ Wahrgenommene Risiken im Zusammenhang mit dem Klimawandel und Anteil der Befragten, die diese spontan benannt haben, in Prozent. Wörtliche Antworten der Befragten wurden anhand übereinstimmender Merkmale in Antwortkategorien zusammengefasst. Dargestellt sind Kategorien, die von mindestens $10 \%$ der Befragten genannt wurden. Von allen Befragten wurden jeweils bis zu fünf Antworten erfasst allergien. Andere Themen wie Hautkrebs oder Sonnenbrand werden zwar mit dem Klimawandel in Verbindung gebracht, dies aber zum Teil wegen einer Assoziation des Themenbereiches Klimawandel mit der Zerstörung der Ozonschicht [32, 35, 36]. Kommunikationskonzepte, die bestehende Vorstellungen aktiv aufgreifen und zu einem besseren Verständnis gesundheitlicher Risiken im Zusammenhang mit dem Klimawandel beitragen, sind somit notwendig.

\section{Von der Risikowahrnehmung zur Handlung}

Welche Herausforderungen für die Risikokommunikation stellen sich aber, wenn Personen bereits überzeugt sind, dass der Klimawandel real und mit Risiken für ihre Gesundheit verbunden ist? Aus der Literatur ist bekannt, dass diese Überzeugung nicht zwangsläufig mit der Intention einhergehen muss, etwas gegen den Klimawandel zu unternehmen bzw. sich aktiv vor den damit verbundenen Risiken zu schützen [37]. Auch in der hier vorliegenden Umfrage unter den Personen, die den Klimawandel nicht verneinen, haben weniger als die Hälfte (46\%) der Aussage zugestimmt, dass sie persönlich zur Eindämmung des Klimawandels beitragen können ( $25 \%$ haben der Aussage voll und ganz zugestimmt). Dies stellt eine Herausforderung für solche Kommunikationsmaßnahmen dar, die eine Anregung zum Handeln und zum aktiven Schutz vor Risiken zum Ziel haben.
Hinzu kommt, dass Kommunikationsmaßnahmen zur Steigerung der Risikowahrnehmung einen negativen Effekt auf die Handlungsbereitschaft haben können. So sind Kommunikationsmaßnahmen zum Thema Klimawandel häufig von furchteinflößenden Szenarien und alarmistischen Appellen geprägt. Ein Beispiel hierfür ist die welt.de-Schlagzeile: „Das Ende der Welt, wie wir sie kennen“, untermalt mit einem dramatischen Gewitterfoto, zum Beitrag über den im Oktober 2018 veröffentlichten Sonderbericht des Weltklimarates [38]. Dass Furchtappelle zwar die Aufmerksamkeit auf den Klimawandel lenken, jedoch gleichzeitig zur Resignation oder gar einer Abwehrhaltung führen können, wird seit Längerem diskutiert [39, 40]. Besorgnis wird nicht automatisch in Handlungen übersetzt $[41,42]$, weshalb reine Furchtappelle bei Personen mit einer bereits ausgeprägten Risikowahrnehmung als wenig zielführend anzusehen sind. Um eine aktive Auseinandersetzung mit dem Thema Klimawandel zu unterstützen und die Bereitschaft $\mathrm{zu}$ Vorsorgemaßnahmen zu fördern, sollte furchteinflößende Kommunikation nur in Maßen eingesetzt werden. Sie sollte zudem immer mit konkreten Handlungsvorschlägen und mit Themen verbunden werden, die Menschen in ihrem Alltag bewegen (z. B. die Gesundheit ihrer Kinder; [39, 40]). Da konkrete Handlungspläne und die Überzeugung, diese umsetzen zu können, die Überführung von Intentionen in Gesundheitsverhalten fördern können [43], sollte der Fokus auf planbare und pragmatische Handlungsmaßnahmen gesetzt werden.

Weitere Faktoren, welche bei der Umsetzung von Risikowahrnehmung in vorsorgende Maßnahmen eine Rolle spielen, sind die Überzeugung, dass eigene und kollektive Maßnahmen überhaupt zur Minderung des Problems beitragen können, sowie die Zuschreibung von Verantwortung. Auch generelle Unsicherheiten in Bezug auf den Klimawandel können die Motivation zu handeln mindern, wenn Zweifel auf die Maßnahmen zu seiner Eindämmung übertragen werden [20, 28]. Gleichzeitig kann die Erwartung, dass andere gesellschaftliche Akteure die Verantwortung für erforderliche Maßnahmen tragen, mit einer geringeren Bereitschaft zum eigenen Handeln verbunden sein [37]. Eine Reihe von Studien deutet darauf hin, dass kollektive, durch die Politik initiierte Maßnahmen (z.B. Erstellung von Hitzeaktionsplänen) individuellen Maßnahmen vorgezogen werden bzw. eine Kombination kollektiver und individueller Strategien (z.B. Ausbau sowie Nutzung eines klimafreundlichen, öffentlichen Personennahverkehrs) befürwortet wird [41, 44]. Kommunikationsmaßnahmen, welche auf individuelle Vorsorgemaßnahmen abzielen, sollten diese idealerweise in ein übergeordnetes Maßnahmenpaket einbetten und in einen Zusammenhang mit lokalen, regionalen oder globalen Strategien stellen.

Eine Einbindung individueller Handlungen in größere soziale, räumliche oder zeitliche Zusammenhänge kann auch die Aufrechterhaltung der ergriffenen Maßnahmen befördern. Maßnah- 
men, die individuell zum Schutz der Gesundheit oder gar zur Eindämmung des Klimawandels ergriffen werden können, werden in der Regel nicht direkt bzw. mit keiner sichtbaren Wirkung belohnt. Stattdessen können sie mit direkt spürbaren Kosten verbunden sein. Dazu zählen beispielsweise finanzielle Kosten wie der Kauf klimafreundlicher, aber teurer Produkte, psychologische Kosten wie der Verzicht auf liebgewonnene Gewohnheiten oder soziale Kosten wie die Ablehnung im sozialen Umfeld [37]. Diese Aspekte stellen eine nicht zu unterschätzende Hürde für das Ergreifen und Aufrechterhalten individueller Maßnahmen dar [20, 37]. Ziel einer gelungenen Risikokommunikation sollte es daher sein, die Vorteile bestimmter Verhaltensweisen und ihre kumulierte Wirkung (z.B. über eine Zeitspanne hinweg) aufzuzeigen. Die Wirkungen verschiedener Maßnahmenoptionen zu veranschaulichen, kann auch die Motivation steigern, wirkungsvolle Alternativen zu wählen. So werden häufig vergleichsweise einfache, aber wenig wirkungsvolle Maßnahmen als Legitimation genutzt, um auf wirkungsvollere, aber aufwendigere Maßnahmen zu verzichten, was in der Literatur als „Tokenism“ bezeichnet wird $[37,45]$. Auch werden Maßnahmen mit positiven Effekten als Berechtigung für solche mit negativen Effekten genutzt (z.B. Reisen mit Flugzeug statt Bahn, da man im Alltag schon auf ein Auto verzichtet), was als „Reboundeffekt“ bekannt ist [37, 45]. Die Veranschaulichung und Kontextualisierung der Effekte ausgewählter Maßnahmen könnte diesen Phänomenen entgegenwirken.

\section{Fazit}

Dauerhafte Änderungen globaler, regionaler und lokaler klimatischer Bedingungen erfordern gezielte Maßnahmen der Risikokommunikation. Diese sollten nicht nur zur Informationsvermittlung über gesundheitliche Risiken dienen, sondern die Bevölkerung mit konkret auf ihre Bedürfnisse abgestimmten Maßnahmen dazu befähigen, auf veränderte Bedingungen $\mathrm{zu}$ reagieren und nötige Handlungsmaßnahmen zu ergreifen. Zum Aufbau dieser „Preparedness“ be- darf es nicht nur eines umfassenden Informationsangebots, sondern auch der Entwicklung von Anleitungen und Handlungsplänen, wie dieses Wissen konkret umgesetzt werden kann. Bei der Entwicklung der entsprechenden Risikokommunikationsmaßnahmen müssen Herausforderungen, die sich aus der Komplexität des Themas und seiner Wahrnehmung in der Bevölkerung ergeben, berücksichtigt werden. Beispiele hierfür sind die psychologische Distanz der Bevölkerung gegenüber dem Klimawandel oder die wissenschaftlichen Unsicherheiten in der Klimaforschung. Die Maßnahmen sollten in ein strategisches Kommunikationskonzept eingebettet sein, welches die Ansprache unterschiedlicher Zielgruppen berücksichtigt.

\section{Korrespondenzadresse}

\section{Natalie Berger, PhD}

Abteilung Risikokommunikation, Bundesinstitut für Risikobewertung (BfR)

Max-Dohrn-Str. 8-10, 10589 Berlin,

Deutschland

natalie.berger@bfr.bund.de

\section{Dr. Ann-Kathrin Lindemann}

Abteilung Risikokommunikation, Bundesinstitut für Risikobewertung (BfR)

Max-Dohrn-Str. 8-10, 10589 Berlin,

Deutschland

ann-kathrin.lindemann@bfr.bund.de

Danksagung. Die Autorinnen danken Eve Gerecke für ihre Unterstützung bei der Literaturrecherche zu diesem Artikel.

\section{Einhaltung ethischer Richtlinien}

Interessenkonflikt. N. Berger, A.-K. Lindemann und G.-F. Böl geben an, dass kein Interessenkonflikt besteht.

Für diesen Beitrag wurden von den Autoren keine Studien an Menschen oder Tieren durchgeführt. Die Befragungsdaten wurden anonym und unter Einhaltung ethischer Richtlinien erhoben.

Open Access Dieser Artikel wird unter der Creative Commons Namensnennung 4.0 International Lizenz (http://creativecommons.org/licenses/by/4.0/deed. de) veröffentlicht, welche die Nutzung, Vervielfältigung, Bearbeitung, Verbreitung und Wiedergabe in jeglichem Medium und Format erlaubt, sofern Sie den/die ursprünglichen Autor(en) und die Quelle ordnungsgemäßnennen, einen Linkzur Creative Commons Lizenz beifügen und angeben, ob Änderungen vorgenommen wurden.

\section{Literatur}

1. Stürzenhofecker M, Zacharakis Z (2018) Die schlechteste Ernte des Jahrhunderts. https:// www.zeit.de/wirtschaft/2018-07/landwirtschaftdeutschland-bauern-ernteausfaelle-duerretrockenheit. Zugegriffen: 17. Sept. 2018

2. Zoronjić S (2018) Hitzewelle 2018: Ventilatoren werden zur Mangelware. https://www.bento. de/today/hitzewelle-2018-ventilatoren-werdenzur-mangelware-a-00000000-0003-0001-0000000002703380.Zugegriffen: 8. Okt. 2018

3. Kindermann K, Kammerinke E (2018) Hitzewelle: Speiseeis-Industriefährt Sonderschichten. https:// www.deutschlandfunk.de/hitzewelle-speiseeisindustrie-faehrt-sonderschichten.766.de.html? dram:article_id=424470. Zugegriffen: 22 . Okt. 2018

4. Schwanke K (2018) Ja, das war ein Jahrhundert-Sommer. https://www.zeit.de/ wissen/umwelt/2018-08/wetter-sommerjahrhundertsommer-karsten-schwankemeteorologie-rekordsommer-deutschland/ komplettansicht.Zugegriffen: 17. Sept. 2018

5. Wecker K (2018) Ist der Klimawandel schuld an der Hitzewelle? https://www.dw.com/de/istder-klimawandel-schuld-an-der-hitzewelle/a44892935.Zugegriffen: 17. Sept. 2018

6. Kovats RS, Valentini R, Bouwer LM et al (2014) Europe. In: Barros VR, Field CB, Dokken DJ et al (Hrsg) Climate change 2014: Impacts, adaption, and vulnerability. Part B: Regional aspects. Contribution of working group II to the fifth assessment report of the Intergovernmental Panel on Climate Change. Cambridge University Press, Cambridge, S1267-1326

7. Eis D, Helm D, Laußmann D, Stark K (2010) Klimawandel und Gesundheit - ein Sachstandsbericht. Robert Koch-Institut, Berlin

8. Spiegel Online (2017) USA erklären Austritt aus Pariser Klimavertrag. http://www.spiegel.de/ politik/ausland/trump-regierung-usa-erklaerenaustritt-aus-pariser-klimavertrag-a- 1161486. html.Zugegriffen: 1. Nov. 2018

9. Farrell J (2015) Network structure and influence of the climate change counter-movement. Nat Clim Chang 6:370

10. Farrell J (2015) Corporate funding and ideological polarization about climate change. Proc Natl Acad Sci 113(1):201509433. https://doi.org/10.1073/ pnas.1509433112

11. Keim ME (2008) Building human resilience. Am J Prev Med 35:508-516

12. Hoegh-Guldberg O, Jacob D, Taylor M, Bindi M, Brown S, Camilloni I, Diedhiou A, Djalante R, Ebi K, Engelbrecht F, Guiot J, Hijioka Y, Mehrotra S, Payne A, Seneviratne SI, Thomas A, Warren R, Zhou $\mathrm{G}$ (2018) Impacts of $1.5^{\circ} \mathrm{C}$ global warming on natural and human systems. In: Masson-Delmotte V, Zhai P, Pörtner HO, Roberts D, Skea J, Shukla PR, Pirani A, Moufouma-Okia W, Péan C, Pidcock R, Connors S, Matthews JBR, Chen Y, Zhou X, Gomis MI, Lonnoy E, Maycock T, Tignor M, Waterfield T (Hrsg) Global warming of $1.5^{\circ} \mathrm{C}$. An IPCC special report on the impacts of global warming of $1.5^{\circ} \mathrm{C}$ above pre-industrial levels and related global greenhouse gas emission pathways, in the context of strengthening the global response to the threat of climate change, sustainable development, and efforts to eradicate poverty. https://www.ipcc. ch/site/assets/uploads/sites/2/2019/02/SR15_ Chapter3_Low_Res.pdf

13. Mücke H-G, Straff W, Faber M et al (2013) Klimawandel und Gesundheit. Allgemeiner Rahmen 
zu Handlungsempfehlungen für Behörden und weitere Akteure in Deutschland. Robert KochInstitut und Umweltbundesamt, Berlin

14. Bunz M, Mücke H-G (2017) Klimawandel - physische und psychische Folgen. Bundesgesundheitsblatt Gesundheitsforschung Gesundheitsschutz 60:632-639

15. Bundesinstitut für Risikobewertung (Hrsg) (2013) Pyrrolizidinalkaloide in Kräutertees und Tees. Stellungnahme 018/2013 des BfR vom 5 . Juli 2013. https://www.bfr.bund.de/cm/343/ pyrrolizidinalkaloide-in-kraeutertees-und-tees. pdf.Zugegriffen: 22. Okt. 2018

16. Stang C, Schwander M (2015) Bekämpfung des Eichenprozessionsspinners (Thaumetopoea processionea) zum Schutz der menschlichen Gesundheit im öffentlichen Raum. UMID Umwelt Mensch Informationsd 27:7

17. Stark K, Niedrig M, Biederbick W, MerkertH, Hacker J (2009) Die Auswirkungen des Klimawandels. Bundesgesundheitsblatt Gesundheitsforschung Gesundheitsschutz 52:699-714

18. Bund/Länder Ad-hoc Arbeitsgruppe Gesundheitliche Anpassung an die Folgen des Klimawandels (GAK) (2017) Handlungsempfehlungen für die Erstellung von Hitzeaktionsplänen zum Schutz der menschlichen Gesundheit. Bundesgesundheitsblatt Gesundheitsforschung Gesundheitsschutz 60:662-672

19. Freter H (2016) Identifikation und Analyse von Zielgruppen in der strategischen Kommunikation. In: Bruhn M, Esch F-R, Langner T (Hrsg) Handbuch Strategische Kommunikation. Grundlagen Innovative Ansätze - Praktische Umsetzung. Springer Gabler, Wiesbaden, S311-325

20. Moser SC (2009) Communicating climate change: history, challenges, process and future directions. Wires Clim Chang 1:31-53

21. Fielding KS, Hornsey MJ, Swim JK (2014) Developing a social psychology of climate change. Eur J SocPsychol 44:413-420

22. McCright AM, DunlapRE(2000) Challenging global warming as a social problem: an analysis of the conservative movement's counter-claims. Soc Probl 47:499-522

23. Davidson MD (2008) Parallels in reactionary argumentation in the US congressional debates on the abolition of slavery and the Kyoto Protocol. Clim Change 86:67-82

24. Michaels D, Monforton C (2005) Manufacturing uncertainty: contested science and the protection of the public's health and environment. Am JPublic Health 95:S39-S48

25. Ding D, Maibach EW, Zhao X, Roser-Renouf C, Leiserowitz A (2011) Support for climate policy and societal action are linked to perceptions about scientific agreement. Nat Clim Chang 1:462

26. Lewandowsky S, Gignac GE, Vaughan S (2012) The pivotal role of perceived scientific consensus in acceptance of science. Nat Clim Chang 3:399

27. McDonald RI, Chai HY, Newell BR (2015) Personal experience and the "psychological distance" of climate change: an integrative review. J Environ Psychol 44:109-118

28. Spence A, Poortinga W, Pidgeon N (2012) The psychological distance of climate change. Risk Anal 32:957-972

29. Trope Y, Liberman N (2010) Construal-level theory of psychological distance. Psychol Rev 117:440-463

30. Abrahamson V, Wolf J, Lorenzoni I et al (2009) Perceptions of heatwave risks to health: Interviewbased study of older people in London and Norwich, UK. J Public Health 31:119-126
31. Grothmann T, Becker R (2015) Der Hitzeknigge. Über das richtige Verhalten bei Hitze. Umweltbundesamt, Dessau-Roßlau

32. Akerlof K, DeBono R, Berry P et al (2010) Public perceptions of climate change as a human health risk: surveys of the United States, Canada and Malta. Int JEnviron Res Public Health 7:2559

33. Leiserowitz AA (2005) American risk perceptions: is climate change dangerous? Risk Anal 25:1433-1442

34. McMichael AJ, Campbell-Lendrum D, Kovats S et al (2004) Global climate change. In: Ezzati M, Lopez AD, Rodgers A, Murray CJL (Hrsg) Comparative quantification of health risks: global and regional burden of disease attributable to selected major risk factors. World Health Organization, Geneva, S 1543-1650

35. Theissen KM (2011) What do U.S. students know about climate change? Eos 92:477-478

36. Bostrom A, Morgan MG, Fischhoff B, Read D (1994) What do people know about global climate change? 1. Mental models. Risk Anal 14:959-970

37. Gifford R (2011) The dragons of inaction: psychological barriers that limit climate change mitigation and adaptation. Am Psychol 66:290-302

38. Brech SM (2018) Das Ende der Welt, wie wir sie kennen. https://www.welt.de/wissenschaft/ article181793882/IPCC-Sonderbericht-zumKlimawandel-CO2-Ausstoss-muss-bis-2050-beinull-liegen.html.Zugegriffen: 19. Okt. 2018

39. O'Neill S, Nicholson-Cole S (2009) "Fear won't dot it": promoting positive engagement with climate change through visual and iconic representations. Sci Commun 30:355-379

40. Moser SC, Dilling L (2011) Communicating climate change: closing the science-action gap. In: The Oxford handbook of climate change and society, $S$ 161-174

41. Lorenzoni I, Nicholson-Cole S, Whitmarsh L (2007) Barriers perceived to engaging with climate change among the UK public and their policy implications. Glob Environ Change 17:445-459

42. Stoll-Kleemann S, O'Riordan T, Jaeger CC (2001) The psychology of denial concerning climate mitigation measures: evidence from Swiss focus groups. Glob Environ Change 11:107-117

43. Schwarzer R, Luszczynska A (2008) How to overcome health-compromising behaviors. Eur Psychol 13:141-151

44. Tvinnereim E, Fløttum K, Gjerstad $\emptyset$, Johannesson MP, Nordø ÅD (2017) Citizens' preferences for tackling climate change: quantitative and qualitative analyses of their freely formulated solutions. Glob Environ Change 46:34-41

45. Gifford RD, Chen AKS (2017) Why aren't we taking action? Psychological barriers to climate-positive food choices. Clim Change 140:165-178 